\title{
Digital chaotic output from an optically processing element
}

\author{
Ana González-Marcos* \\ José A. Martín-Pereda, MEMBER SPIE \\ Universidad Politécnica de Madrid \\ E.T.S. Ingenieros de Telecomunicación \\ Ciudad Universitaria \\ 28040 Madrid, Spain \\ E-mail: jamp@tfo.upm.es
}

\begin{abstract}
Digital chaotic behavior in an optically processing element is reported. It is obtained as the result of processing two fixed trains of bits. The process is performed with an optically programmable logic gate, previously reported as a possible main block for optical computing. Outputs for some specific conditions of the circuit are given. Digital chaos is obtained using a feedback configuration. Period doublings in a Feigenbaum-like scenario are obtained. A new method to characterize this type of digital chaos is reported. (C) 1996 Society of Photo-Optical Instrumentation Engineers.
\end{abstract}

Subject terms: optical computing; digital chaos; logic cells; period doubling; line encoder.

Paper 16025 received Feb. 15, 1995; revised manuscript received July 3, 1995; accepted for publication July 4, 1995. This paper is a revision of a paper presented at the SPIE conference on Chaos in Communications, July 1993, San Diego, CA. The paper presented there appears (unrefereed) in SPIE Proceedings Vol. 2038.

\section{Introduction}

The dynamic behavior of nonlinear systems have been studied intensively in recent years. Most of the work concerning the temporal operation of such systems has been done with analog signals and on the assumption that nonlinear optical systems are described by differential equations. Indeed, this mathematical model is a good approximation of all systems with time constants large compared to the mean group delay or round-trip time of the feedback loop. Taking into account a finite feedback delay comparable to or greater than the combined time constants of all system components however, the dynamics of the system are given by difference-differential equations. Ikeda ${ }^{1}$ was the first to apply this type of analysis to a ring cavity system with a nonlinear medium. From his results, he concluded that new types of instabilities should be found in such systems yielding periodic and even chaotic solutions. Furthermore, Okada and Takizawa ${ }^{2}$ investigated the effect of a delayed feedback in a hybrid electro-optic system with the restriction that the delay is less than or comparable to the response time of such a system. Finally, Neyer and Voges ${ }^{3}$ studied the pure effect of the feedback delay on the behavior of an electro-optic system, neglecting all time constants of the system components. Several other recent studies have been carried out following these main lines.

The optical structure whose nonlinear behavior is reported here is a configuration we reported ${ }^{4-6}$ previously. It is able to process two input binary signals, and its two outputs are logical functions of these inputs. The type of processing is related to the eight main Boolean functions,

\footnotetext{
*Current affiliation: Universidad Alfonso X el Sabio, Villanueva de la Canada, Madrid, Spain.
}

namely, AND, OR, XOR, NAND, NOR, XNOR, ON, and OFF. The programmable ability of the two outputs, as described in the first part of this paper, enables the generation of several-data coding for optical transmission.

Moreover, the main objective of this paper is to demonstrate the possibility to apply this structure to the generation of periodic and even chaotic solutions. A precise analysis of the output characteristic versus the main variable parameters, such as control signal level and data signal level, has yielded some results that can be described with the help of the chaos theory, which makes it possible to understand the behavior reported here.

\section{Optical Configuration of the Optically Programmable Digital Circuit}

The considered optically programmable digital circuit has been already reported ${ }^{4-6}$ as a programmable logic gate. Its working characteristics, and the way it has been practically implemented are briefly summarized here. This description is needed to clarify some of the points employed in its present application. A detailed discussion of the different possible devices to be employed and the transmission medium used for its physical implementation can be found in Ref. 6.

A block diagram of the circuit is show in Fig. 1. As can be seen, the circuit is composed by two optical devices $P$ and $Q$ of nonlinear behavior. Their outputs correspond to the two final outputs $O_{1}$ and $O_{2}$ of the cell.

The four possible inputs to the circuit are two for input data, $I_{1}$ and $I_{2}$, and two, $g$ and $h$, for control signals. The way these four inputs are arranged inside the circuit is also represented in Fig. 1. The corresponding inputs to the nonlinear devices, $P$ and $Q$, are functions of these signals plus, 


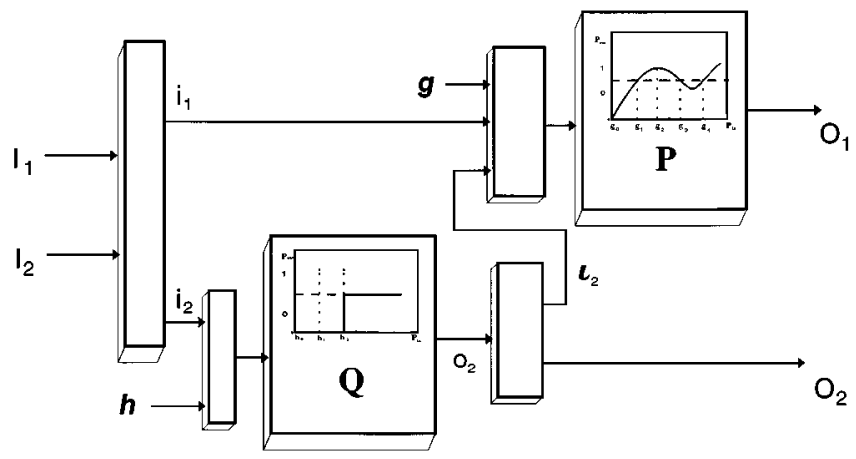

Fig. 1 Block diagram of the optically programmable logic circuit. The open boxes are $2 \times 2$ or $2 \times 1$ couplers; $i_{1}=i_{2}=\left(I_{1}+I_{2}\right) / 2$; $\iota_{2}=O_{2}=O_{2} / 2 ; g$ and $h$ are control signals; and $I_{1}$ and $l_{2}$ are the input data signals.

in the case of the $P$ device, another coming from inside its own cell and obtained from the $Q$ device.

Our practical implementation of the processing element has been based on an optoelectronical configuration. The lines in Fig. 1 represent optical multimode fibers. The indicated blocks, placed to combine the corresponding signals, are conventional optical couplers. In this way, inputs arriving to the individual devices are multilevel signals.

The working levels are

$I_{P}=\left(I_{1}+I_{2}\right) / 2+g+o_{2} / 2$

for device $P$ and

$I_{Q}=\left(I_{1}+I_{2}\right) / 2+h$ for device $Q$. The output from device $Q, o_{2}$, is divided into two equal-intensity signals. One is the final output $\mathrm{O}_{2}$ and the other, $\iota_{1}=o_{2} / 2$, becomes a part of the input to device $P$.

Therefore, the output of the $P$ device depends on the control signal $g$, plus one half of the output $\mathrm{O}_{2}$ of the $Q$ device. The output of the $Q$ device depends only on its control signal $h$. We understand "output" as the type of processing, or logical function, that executes each of the devices on the two binary data inputs.

The characteristics of the nonlinear devices are also shown in Fig. 1. Device $Q$ corresponds to a thresholding or switching device, and device $P$ is a multistate device, where the ideal response of this nonlinear optical device is the one represented in Fig. 1. This response is similar to that achieved by a SEED device. Because the input signal is a multilevel signal, as can be seen from Eqs. (1) and (2), the output depends on the relations among

1. the level of a bit 1

2. the level of the control signal

3. the level for switching from one state to another. This level is intrinsic to the employed device.

To clarify the preceding facts, we have represented in Fig. 2 how one of the possible outputs for each device is obtained. The previously mentioned parameters are indicated in the same figure. Moreover, nonlinear devices $P$ and $Q$ are shown with their ideal shape as adopted. Other logical operations can be obtained in a similar way. These devices were experimentally implemented with an optoelectronic approximation.

In the results to be reported here, however, we have not paid much attention to the experimental implementation but

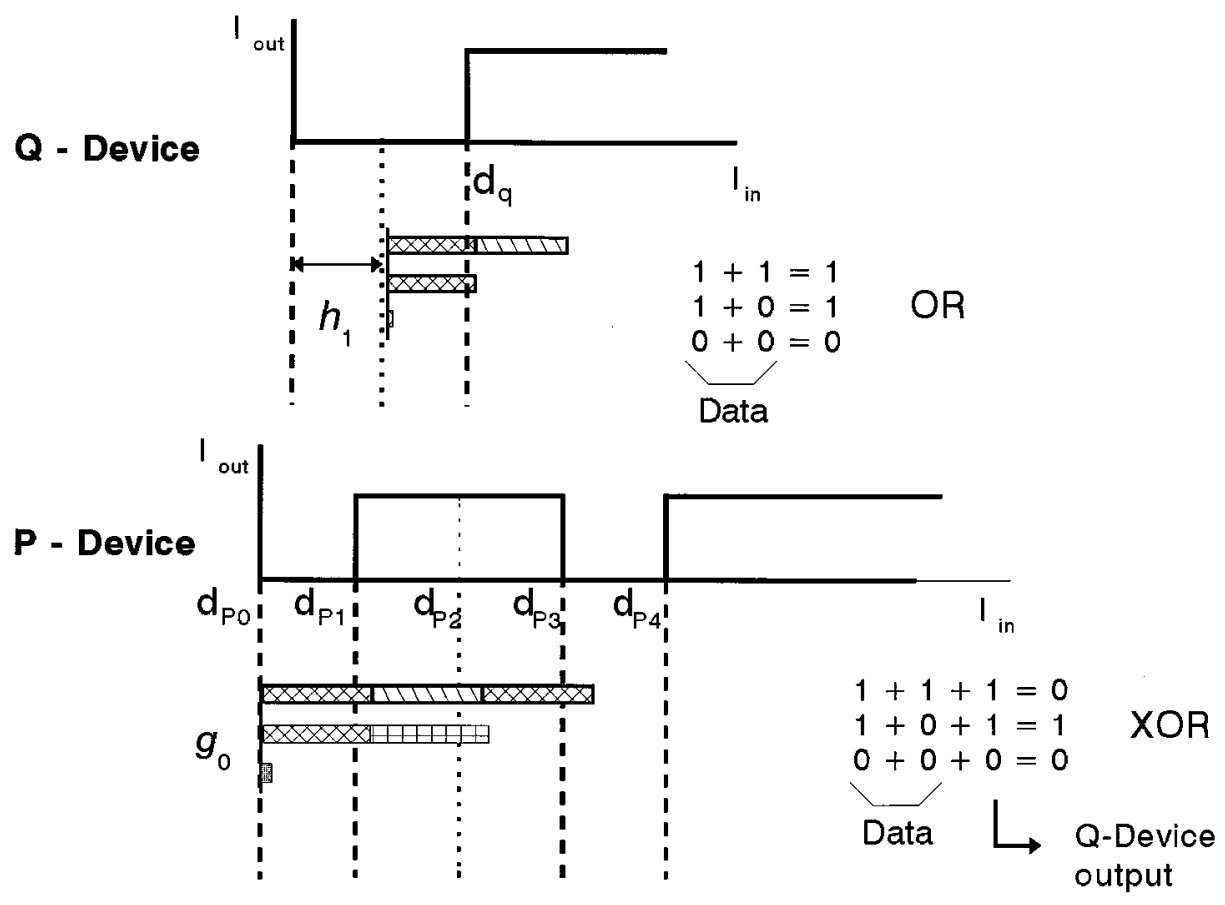

Fig. 2 Example of input/output characteristics for $Q$ and $P$ devices for a particular situation; $d_{P, Q}$ are the corresponding decision levels for $P$ and $Q$ devices. 
Table 1 Output functions of the optically programmable logic circuit.

\begin{tabular}{lccc}
\hline \hline $\begin{array}{l}\text { Control signal } \\
P \text { Control signal }\end{array}$ & $\begin{array}{c}Q \text { Output: AND } \\
0-0.4 \\
P \text { Output }\end{array}$ & $\begin{array}{c}Q \text { Output: OR } \\
0.5-0.9 \\
P \text { Output }\end{array}$ & $\begin{array}{c}Q \text { Output: ON } \\
1.0-2.0 \\
P \text { Output }\end{array}$ \\
\hline $0-0.4$ & XOR & XOR & NAND \\
0.5 & NAND & NOR & NOR \\
$0.6-0.9$ & ON & XNOR & XNOR \\
1.0 & XNOR & XNOR & AND \\
$1.1-1.4$ & XNOR & ON & OR \\
1.5 & AND & OR & OR \\
$1.6-2.0$ & OR & OR & ON \\
$2.0-2.5$ & ON & ON & ON \\
\hline \hline
\end{tabular}

rather to its computer simulation, which was accomplished with the MATLAB ${ }^{\mathrm{TM}}$ program and the SIMULINK ${ }^{\mathrm{TM}}$ application.

The value of a bit 1 at any of the two inputs of the cell, namely $I_{1}$ or $I_{2}$, was considered as a normalization value for the present simulation of the optically programmable logic circuit. The changing parameters are chosen as

1. the decision levels of each device, $d_{Q}$ and $d_{P}$ (see Fig. 2)

2. the controls signals $g$ and $h$.

Moreover, we have considered five decision levels in the $P$ device. These are equidistant with respect to the first one $d_{P 1}$. There are, however, only three input levels where the output is able to switch from one state to another. This fact is in good agreement with the real characteristics of the optical nonlinear device considered, namely, a self-electrooptic-device (SEED).

In a practical situation, and as a first application of the structure, the decision levels for each device have welldefined values. We have fixed them to $d_{Q}=1$ and $d_{P 1}=0.5$.
By changing both control signals $g$ and $h$ from 0 to 2.5, a total of 14 pairs of logic functions can be obtained. These are summarized in Table 1.

Another way to work with this cell is the possibility of controlling the decision levels. These levels are intrinsic to each particular device employed. In our case, and by maintaining the same normalization as before, other different tables, equivalents to Table 1 , can be obtained by changing these decision level values. To show a more general example, we analyze and report only the results corresponding to the output $O_{1}$, which can be seen in Fig. 1, is related to the more complex device $P$. A similar study can be done for the $Q$ device.

The behavior of the $O_{1}$ output for functions $\mathrm{ON}$ or OFF at output $\mathrm{O}_{2}$ is shown in Fig. 3. The axes represent the power level of control signal $h$ on the $y$ axis and, the power level of decision signal $d_{P 1}$ on the $x$ axis.

As we can see, a large variety of logic functions are obtained in a very simple way. Moreover, a particular point needs to be noted concerning the extreme sensitivity of the output logic functions with respect to both control signals and decision levels. This fact is of a great importance for further considerations of the working conditions.

A further point that needs to be considered concerns previous structures with a behavior similar to that of the one reported in this work. A somewhat similar all-optical programmable logic gate was proposed previously. ${ }^{7-9}$ The practical implementation of this gate has shown one of the major problems with the technique of free-air transmission and on-axis addressing: all inputs need to be exactly collinear to produce the same characteristics for each input. Moreover, external conditions can affect to the global behavior from both losses and mechanical stability. None of these problems are present in our system because of the fiber connections between nonlinear devices. Smaller losses in the system is another characteristic of our circuit. Moreover, it can perform arithmetic operations such as 2-bit addition generating its corresponding carry and hence to act

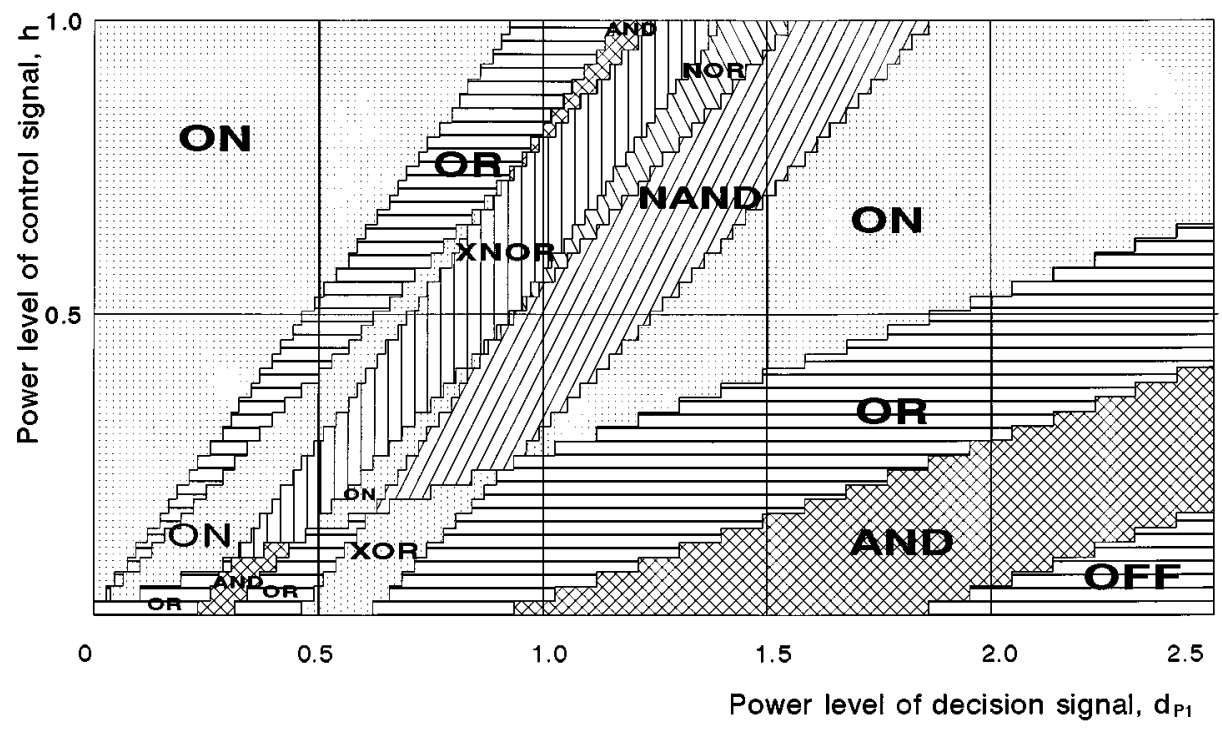

Fig. 3 The $O_{1}$ output behavior of the optically programmable logic circuit for an ON output at gate $O_{2}$. The axes 4 show the power level of the control signal $h$ versus the power lever of the decision signal $d_{P 1}$. Both signals are normalized to an input bit 1 . 


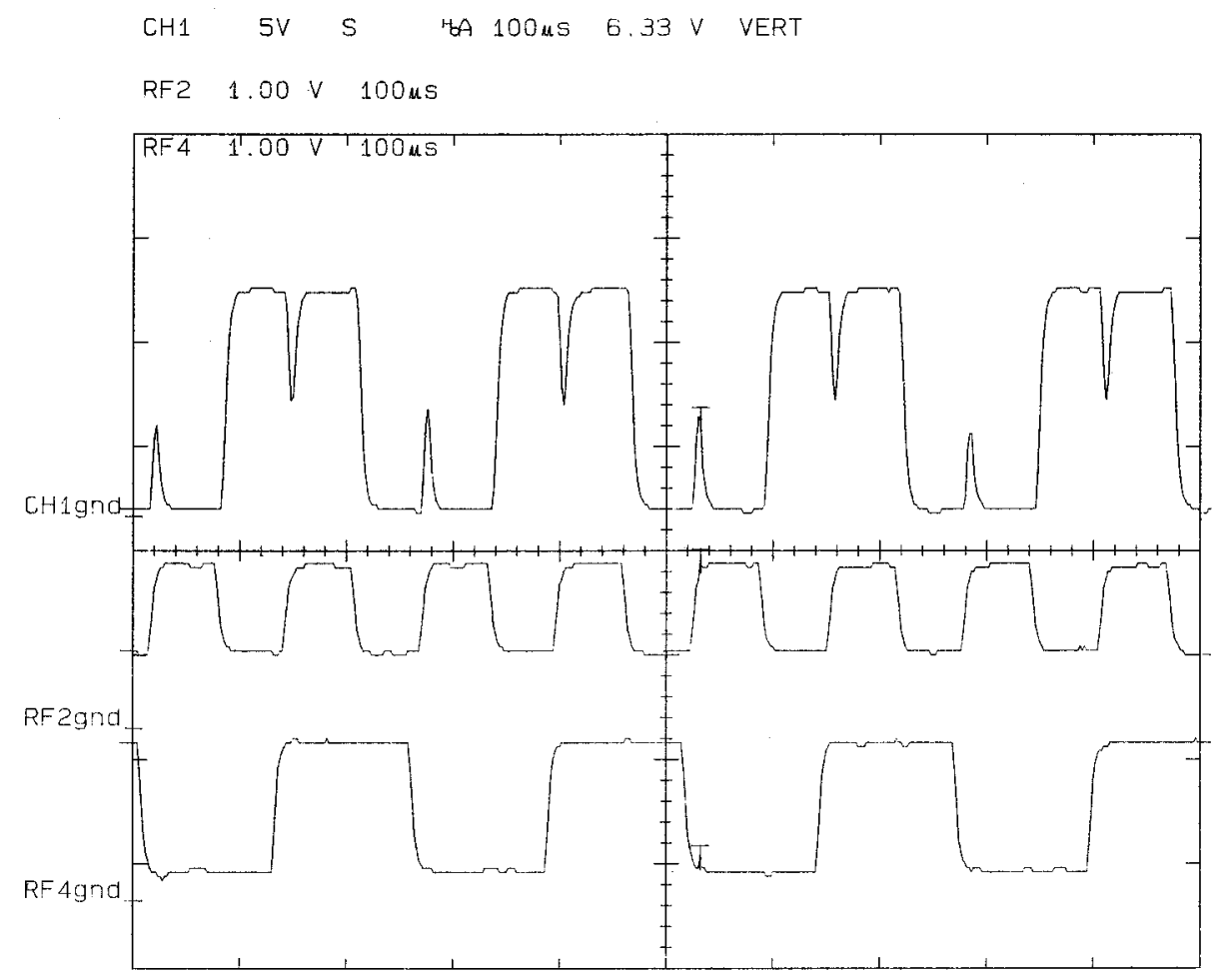

Fig. 4 Manchester encoding from an NRZ data stream. Peaks indicate the beginning of a bit time.

as a ripple carry-adder. ${ }^{6}$ These points, however, are related more to its application in optical computing than to our present approach. More details will be given elsewhere.

\section{Application of the Logical Circuit as a Line Encoder for Optical Communications}

Although the main objective of a logical circuit, such as the previously analyzed one, is for optical computing, there are some other possibilities. Most of these are related to its application to such other fields as optical communications. Two of the more important applications are the related to line encoding and the generation of a periodic pulse sequence from a constant input. The first is presented in this section.

In our work, the discussed structure has been able to perform line coding. As an example, biphase or Manchester encoding is shown, but almost any two-level block codes of the $n B m B$ type can be obtained.

As can be seen in Table 1, one of the possible outputs through port $O_{1}$ is an XOR function of the two inputs. This enables us to obtain a Manchester encoding in a very easy way. If the initial uncoded NRZ data stream is fed to input $I_{1}$ and a train of 0 and 1 alternated bits, with a value of half the period of the previous one, goes to input $I_{2}$, the resulting output in $O_{1}$ is the needed biphase encoding. Our results are shown in Fig. 4 where the initial NRZ data are shown together with the newly obtained data. This result was obtained experimentally.

A view of our cell is shown in Fig. 5. As can be seen, optical interconnections between elements are carried out by optical fibers. The $1 \times 2$ couplers are the mixing signal elements. Because nonlinear all-optical devices with adequately high speed were not available to us, nonlinear de- vices $P$ and $Q$ were simulated by optoelectronic methods. Light coming to them was converted to an electrical signal and the nonlinear optical behavior was simulated by electronic circuitry. The output of the circuit was again a light beam sent to the fiber. Multimode optical fibers were employed and common LEDs were used as light sources. No attempt was given to obtain high bit rates, but only the method validity.

\section{Periodic and Chaotic Behavior}

Nonlinear behavior can be expected from the cell just reported if some kind of feedback is applied. Some examples of this type of behavior are given in Refs. 2 and 3. Although the present configuration has almost no points in

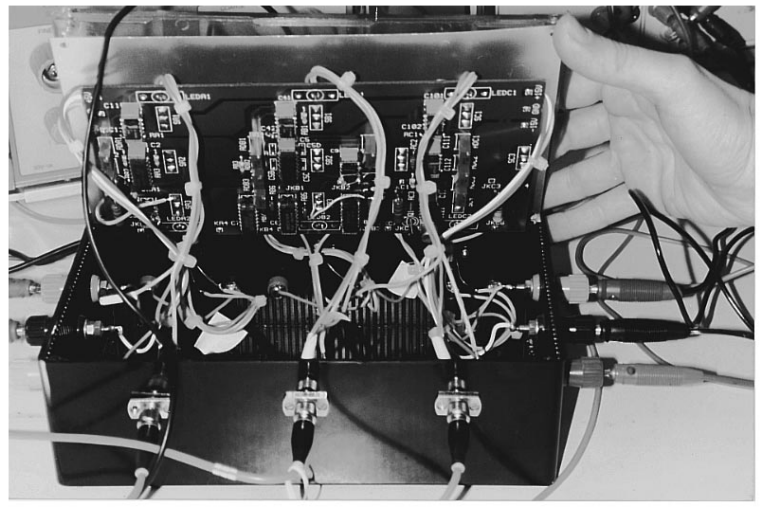

Fig. 5 Close view of the experimental setup. 


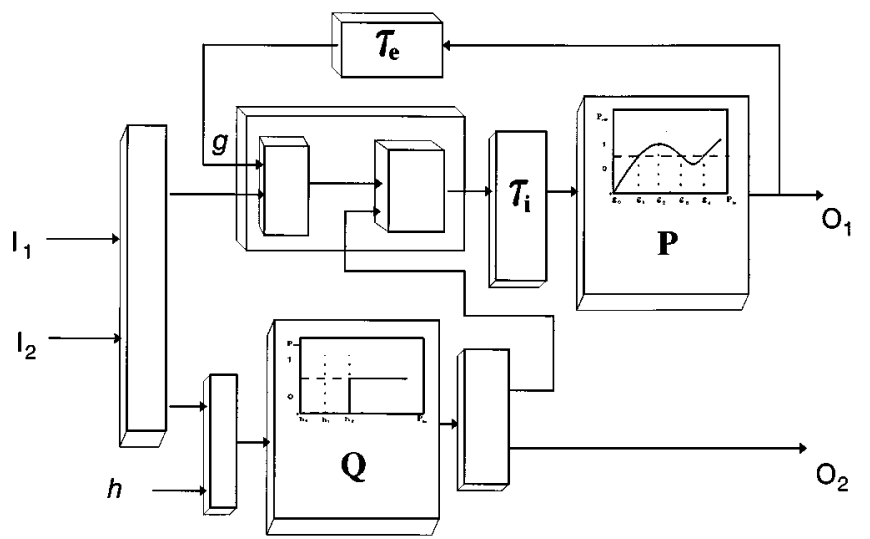

Fig. 6 Optical processing element with feedback; the open boxes are $2 \times 2$ or $2 \times 1$ couplers and $\tau_{e}$ and $\tau_{i}$ are external and internal delay times.

common with the cases reported there, the presence of nonlinear devices in the system has some analogies with them.

To study the nonlinear response of our circuit, some minor modifications are needed. The first is to introduce a feedback from one of the two possible outputs to one or both of the cell inputs. Moreover, according to previous studies in this field, the introduced feedback requires some time delay. In the same way, because our results are obtained by computer simulation, another internal delay is needed. It corresponds to the response time of the simulated nonlinear devices $P$ and $Q$.

In general, a periodic behavior should be expected as the normal output of the system. Under some conditions, however, this is not always true. The output, as we show, is not periodic with some parameter values of the system.

If feedback is to be applied to the system, we have two possibilities, corresponding to the two outputs $O_{1}$ and $O_{2}$. Because, depending on its control signal (see Table 1), the $P$ device output has more possible different functions than that of the $Q$-device, we adopted its output as signal feedback. Again, there were several possible ways to feed this signal to the cell, because there are four possible inputs. The solution adopted was to go to the control input $g$ of $P$ device. No other additional control signal is used. Figure 6 shows the final circuit with feedback.

The first analysis that we have performed using simulation tools considered null delay times. This situation has no algebraic solution and no data were obtained. However, the results are significantly different if finite delay times, namely, internal and external delays, are introduced.

According to previous studies, ${ }^{2,3}$ it is more likely to obtain a periodic or even chaotic solution when the internal delay time is shorter than the external one. In each of our studied cases, a regular train of pulses was the input. The real input to device $P$, before the feedback takes place, is shown in Fig. 7. As can be seen, it is a multilevel signal corresponding to the addition of the two inputs. The period of this signal corresponds to a time of 14 bits.

If the ratio between internal and external delay times is smaller than 1 , we obtain a periodic situation. The period of this signal is strongly dependent on the ratio value. In the case where the external time delay is 200 and internal delays are 2, 4, and 12, the obtained results are as summarized in Fig. 8. As can be seen, a particular string of 0's and 1's is periodically repeated. These results are indicated in Table 2. An interesting result is the duplication in period time when the ratio between delays grows smaller; it goes from 70 to 280 time units. Hence, frequency doubling have been obtained. This is one of the best indications of a possible route to chaos.

A further point must be taken into account. The values given in Table 2 do not correspond to the transition points between different periods. They are in a range where the period remains constant. If we calculate the equivalent to the Feigenbaum ratio for the indicated values we obtain 4. If higher order transition points were considered, however, a number closer to the Feigenbaum number 4.669201... should have been obtained.

Finally, we set the internal response time equal to zero. The input signal corresponding to feedback plus input data, to the $P$ device control gate, is shown in Fig. 9. The output signals are given in Figs. 10(a) through $10(\mathrm{~g})$. No indication of a possible periodic behavior was obtained for longer time intervals. Because they have been achieved after a period-doubling route, these results, clearly indicate that a certain type of chaos is present.

To characterize the obtained chaotic signal, conventional methods are difficult to apply here. The preceding results constitute a time series from which a chaos measure should be obtained. But it is not possible, however, to grasp the correct phase-space representation from these results in an straightforward way. We do not know what the adequate phase-space variables are and it is not known how many

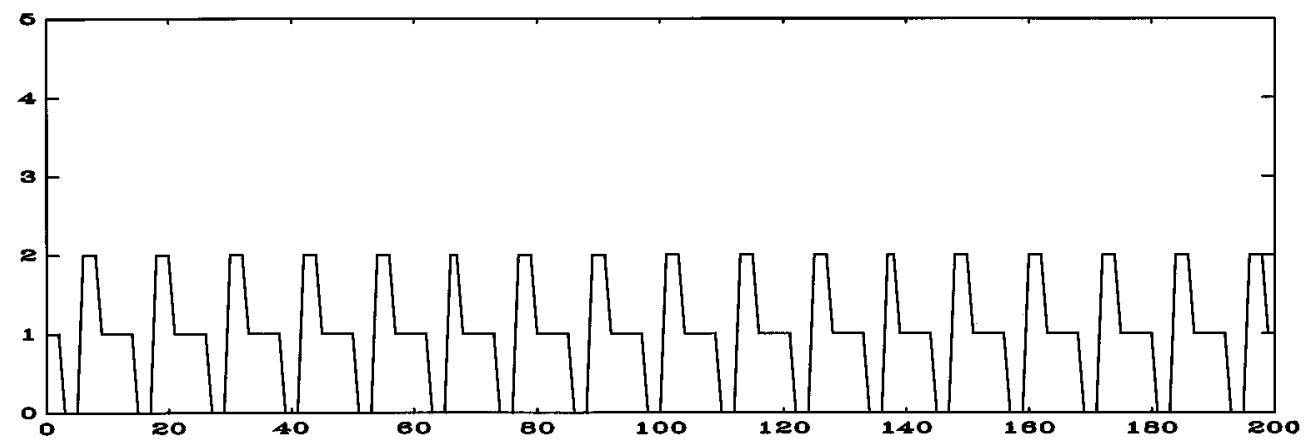

Fig. 7 Input to the $P$ device before feedback. 


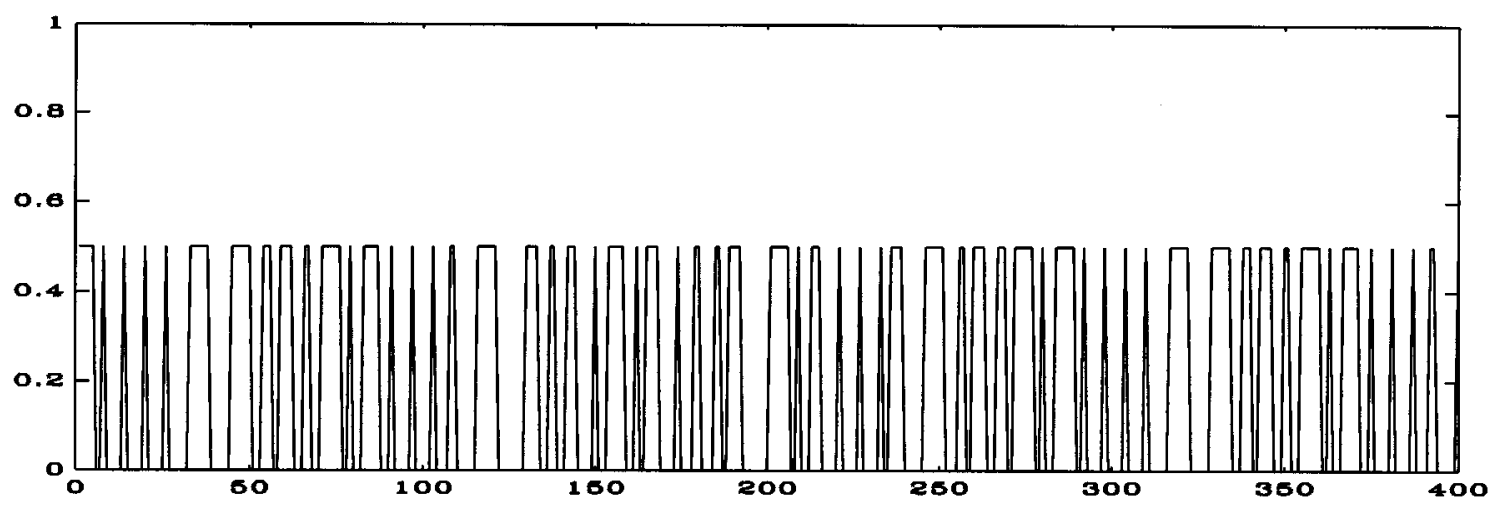

Output signal with a period of 280 .

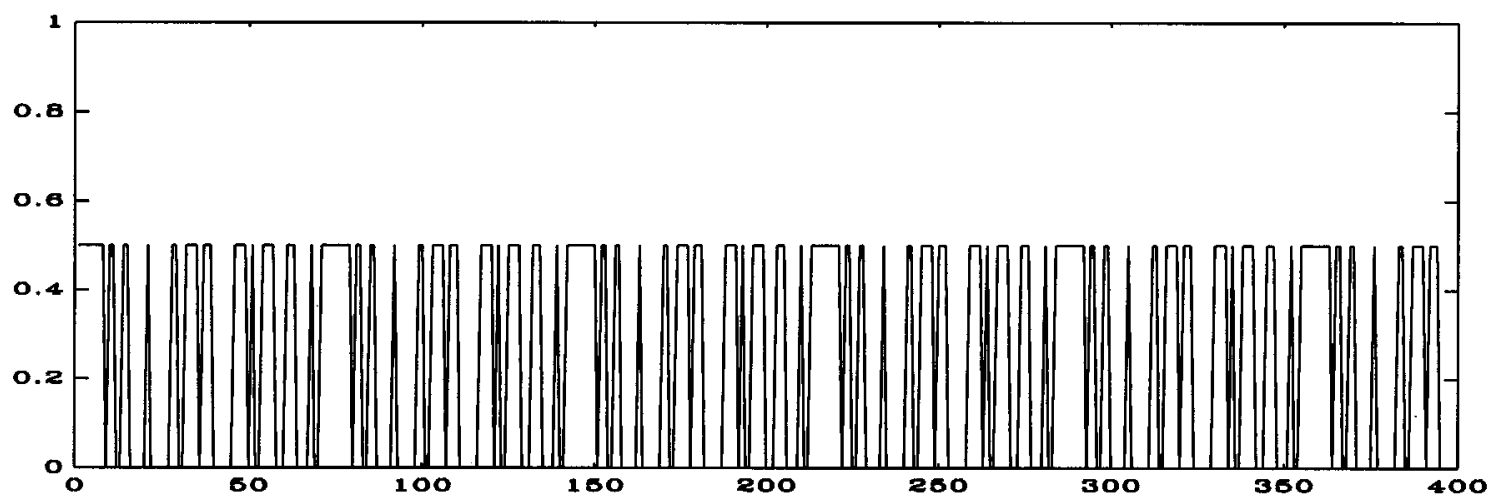

Output signal with a period of 140 .

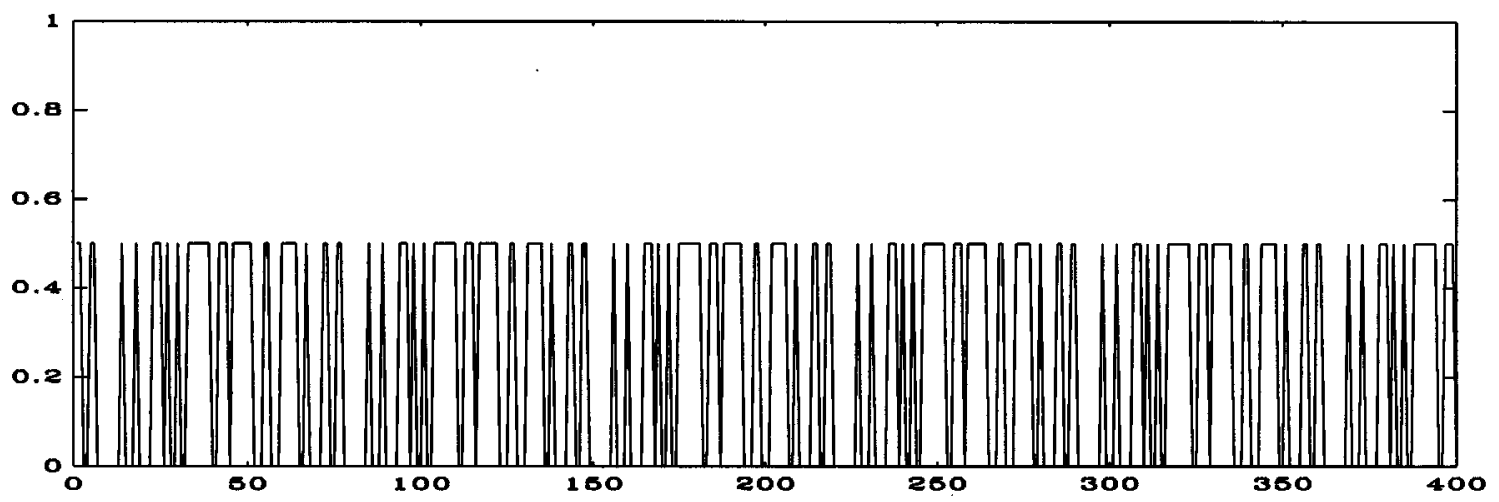

Output signal with a period of 70.

Fig. 8 Periodic output signals when feedback is added. Time periods are indicated at each signal.

variables are needed to fully describe the dynamics of this particular system. Fortunately a partial answer to this problem has been applied successfully in a large number of experimental investigations. The basic idea is that if the fundamental phase-space variables are $x$ and $x^{\prime}$, to study the evolution of the system numerically, $x$ and $x^{\prime}$ must follow as functions of $t$. Because $x^{\prime}=[x(t+\Delta t)-x(t)] / \Delta t$ in the limit as $\Delta t \rightarrow 0$, however, a knowledge of $x(t+\Delta t)$ is equivalent to a knowledge of $x^{\prime}$. In other words, a knowledge of a trajectory of points $[x(t), x(t+\Delta t)]$ is equivalent to a knowledge of the trajectory of points $\left[x(t), x^{\prime}(t)\right]$. As a consequence, the phase-space trajectory

$x(t)=\left[x_{1}(t), x_{2}(t), \ldots, x_{n}(t)\right]$ 
Table 2 Characteristics of the output signals according to the delay times.

\begin{tabular}{rrrrr}
\hline \hline$t_{p}$ & $\tau_{e}$ & $\tau_{i}$ & $\tau_{i} / \tau_{e}$ & Period \\
\hline 14 & 200 & 2 & 0.01 & 280 \\
14 & 200 & 4 & 0.02 & 140 \\
14 & 200 & 12 & 0.06 & 70 \\
\hline \hline
\end{tabular}

is replaced by a trajectory in an artificial phase space with points given by

$y(t)=[y(t), y(t+\Delta t), \ldots, y(t+m \Delta)]$,

where $y(t)$ is any one of the phase-space variables $x_{i}(t)$. Thus, from a set of measurements of a single quantity $y(t)$ we can construct a sequence of points in an artificial phase space

$$
\begin{aligned}
x(t) & =[y(t), y(t+\Delta t), \ldots, y(t+m \Delta t)], \\
x(t+\Delta t) & =[y(t+\Delta t), \ldots, y(t+(m+1) \Delta t)],
\end{aligned}
$$

$$
\vdots \text {. }
$$

With the data we have, the first problem to solve is how to operate with our digital signal where just two values, 0 and 1 , are present. If we adopt only this output as possible values for $y$, the resulting plot at the phase space should be concentrated on just four points, namely, $(0,0),(0,1),(1,0)$, and $(1,1)$. Almost no information could be obtained from this plot. Hence a new technique must be implemented.

The first method we adopted is to group sets of four consecutive bits and to convert them to their corresponding hexadecimal values. Hence, a sequence of zeros and ones is converted to a new string of hexadecimal values, namely, $0,1,2, \ldots, 15$. For example, 0010 would be a 2,1001 a 9 , and 1110 would be a 14 . The total number of data is divided by 4 , but much more information can be obtained than with simple binary signals. A diagram similar to the $t_{i+1}$ versus $t_{i}$ in analogue signals can be drawn in this way. In the case of periodic signals, a closed configuration is obtained. In the case of chaotic signals, however, no definite pattern is obtained. This situation appears in Fig. 11, where the plot for the output signal given in Fig. 10 is shown.

A further point that needs to be considered is the one concerning the justification that the preceding quantity, namely the hexadecimal sequence, represents the same behavior of the system as the previously obtained binary one. But this situation is equivalent to the reverse one: to convert a chaotic analog signal into a digital one. As is well known from digital communications, any analog signal can be quantized and from this quantization to obtain a digital signal with the same main properties than the initial analog one. In our present case, we have accomplished the opposite operation, namely, to convert a digital signal into an analog quantized one with sixteen possible levels. And, according to digital communication signal processing, both representations are equivalent. Moreover, some theoretical aspects of this relation have to be studied in a further work.

A second possible method to study this particular type of chaos is by the Lempel and Ziv (LZ) complexity measure, which is based on extracting meaningful information from data that have every appearance of being random and determining a computable measure of their complexity. One of the main methods is that studied by Lempel and Ziv, ${ }^{10}$ which measures the number of distinct patterns that must be copied to reproduce a given string. The only computer operations considered in constructing a string are copying old patterns and inserting new ones. Briefly described, a string $S=s_{1} s_{2} \ldots s_{n}$ is scanned from left to right, and a complexity counter $c(S)$ is increased by one unit every time a new substring of consecutive digits is encountered in the scanning process. The resultant number $c(S)$ is the complexity measure of the string $S$. According to Ref. 11, the minimum value for LZ complexity is $c(S)=2$ and, moreover, only relative values of $c(S)$ are meaningful and, in particular, it is the comparison with the $c(S)$ value for a random string that is meaningful. Lempel and $\mathrm{Ziv}$ have shown that for a random string of length $n$, the LZ complexity is given by

$b(n)=\frac{h n}{\log _{K}(n)}$,

where $K$ denotes the number of elements in the alphabet $A$ and $h$ denotes the normalized source entropy

$h=\frac{-1}{\log N} \sum_{i=1}^{N} p_{i} \log p_{i} \leqslant 1$.

In our case, each symbol from the alphabet, namely 0 and 1 , is equally probable and hence $p_{i}=1 / N$ and $h=1$.

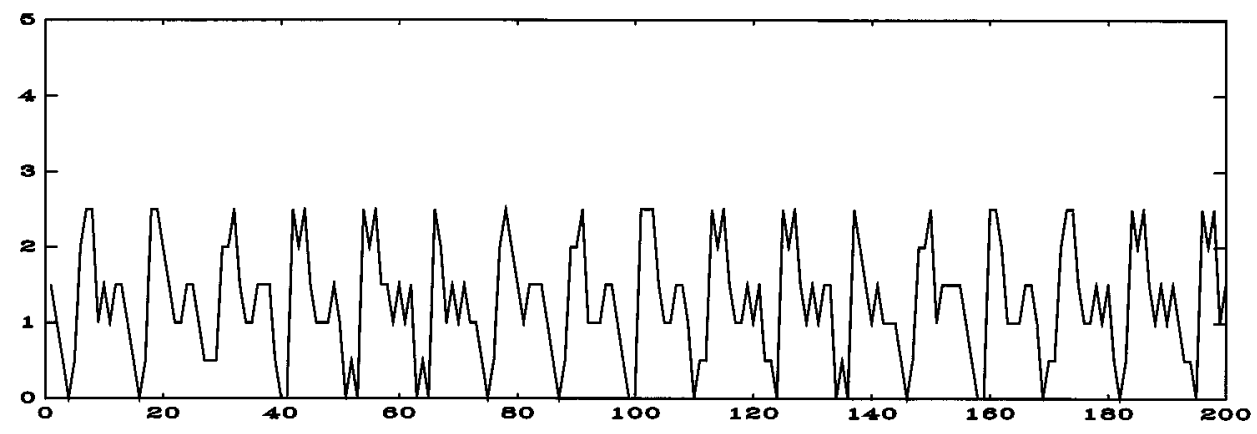

Fig. 9 Input to $P$ device when feedback is present. 


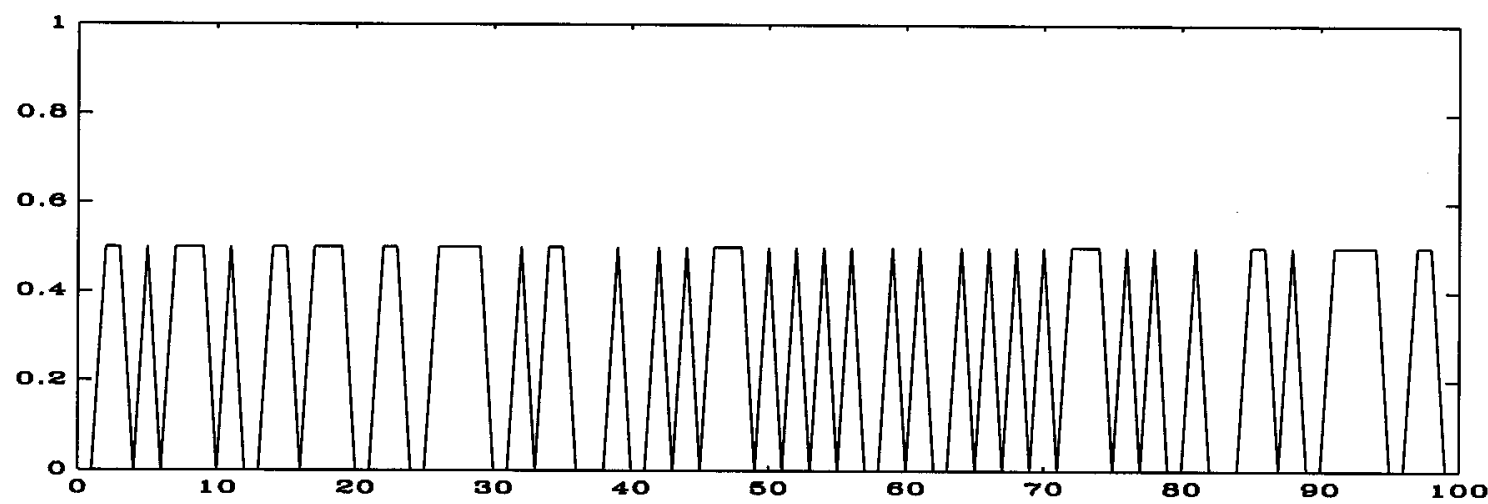

(a)

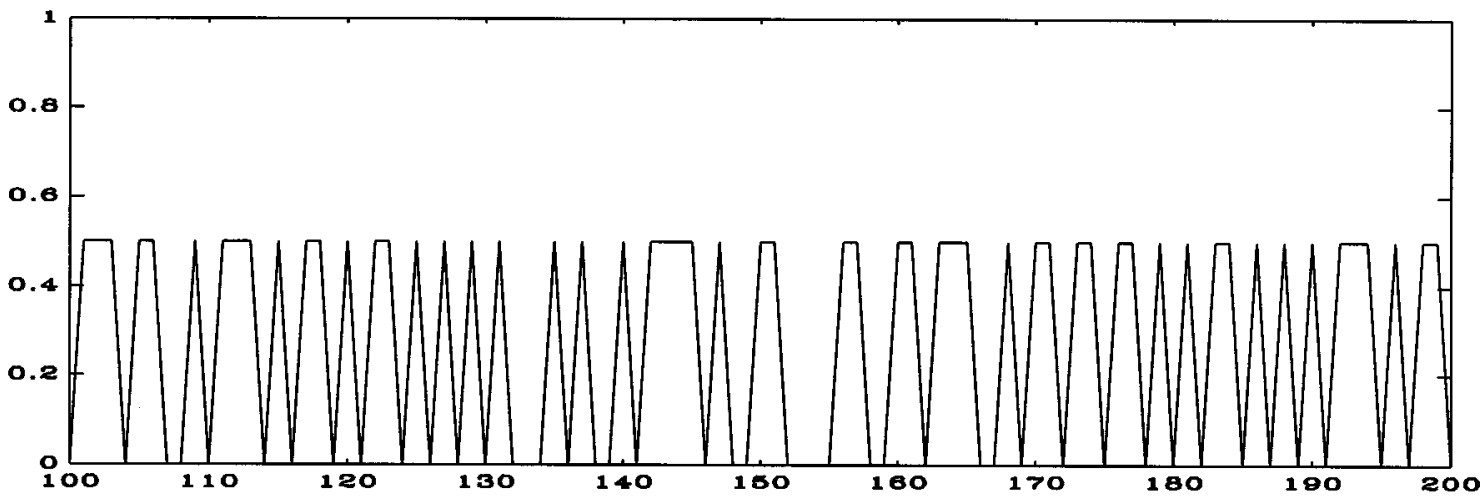

(b)

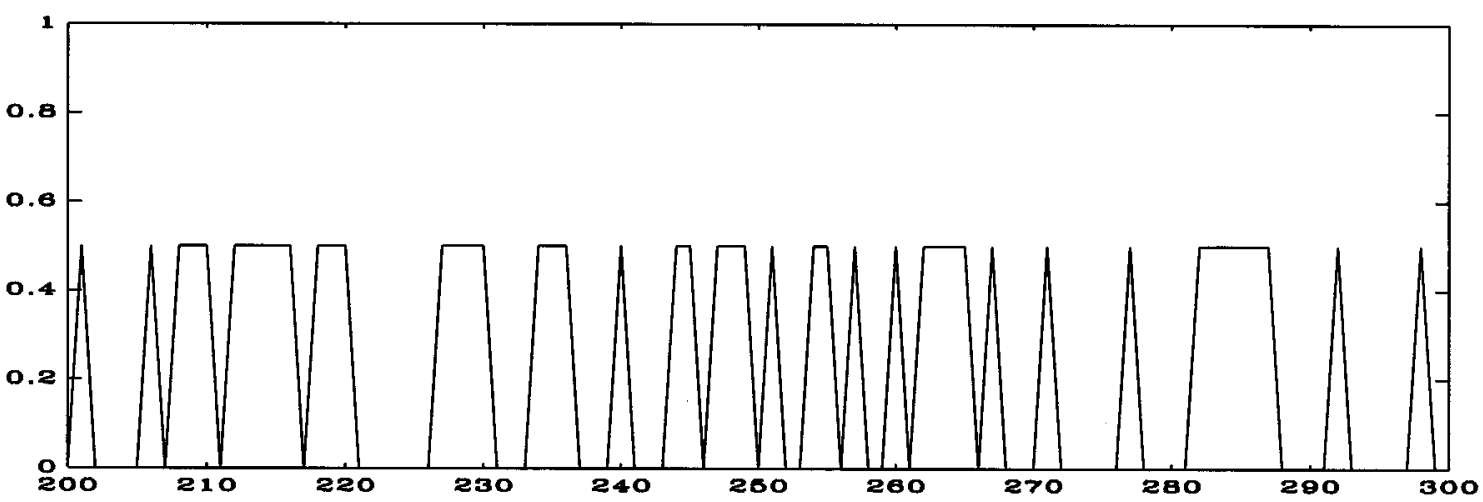

(c)

Fig. 10 Time evolution of output signal when internal delay time is zero. A chaotic behavior is present.

To compare with the complexity for a random string, we must compute

$\lim _{n \rightarrow \infty}[c(S) / b(n)]$

for a string with $n$ elements. If the preceding ratio is less than 1 , then we can conclude that this is caused by a pattern formation in the string $S$.
We have applied this method to the study of the two types of patterns obtained before, namely, periodic and chaotic. In every case, we have taken strings with 2064 digits. The results obtained are summarized in Table 3. As it can be seen, values much smaller than 1 are obtained for those outputs where a periodic pattern was present. On the contrary, a value very close to 1 appears for the chaotic solution. Values should be much more different if larger strings were taken. 


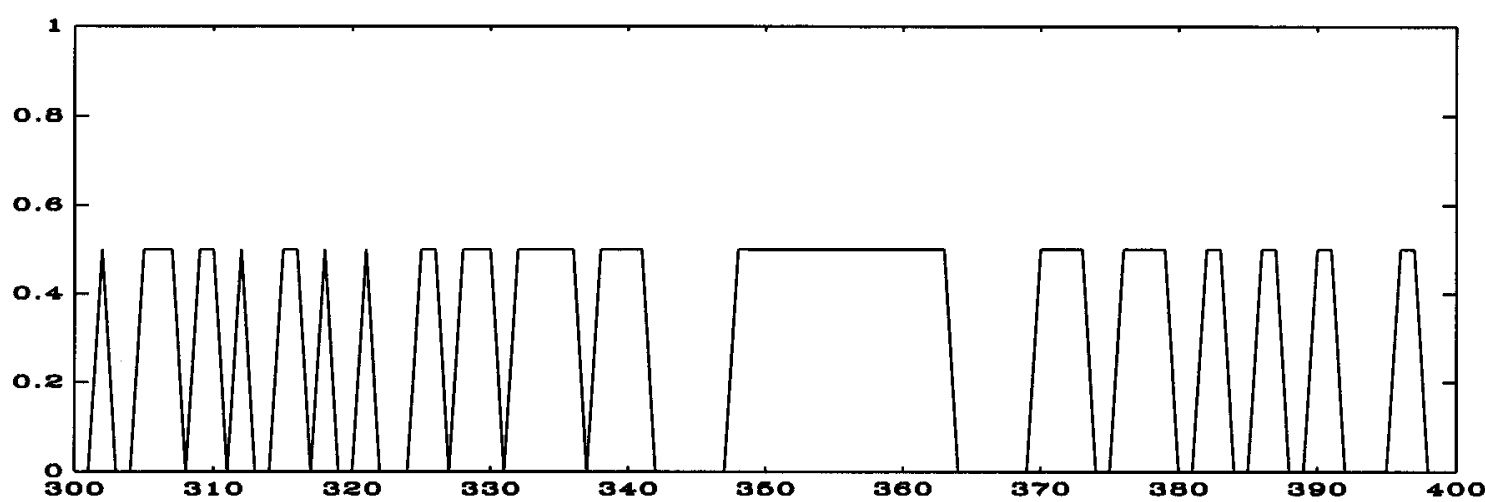

(d)

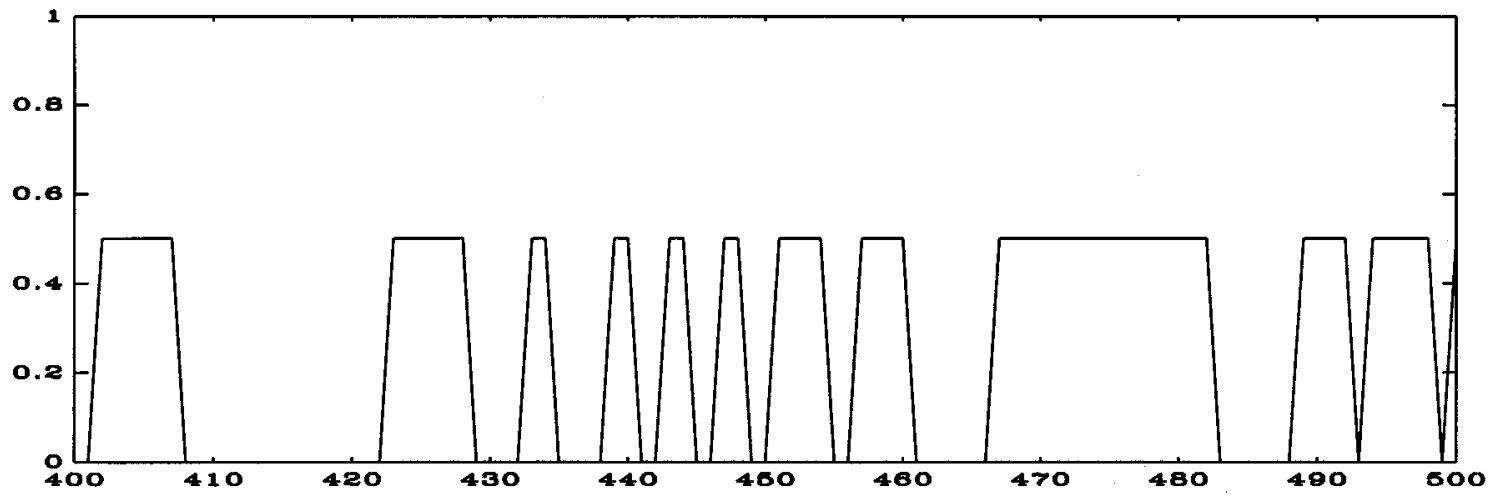

(e)

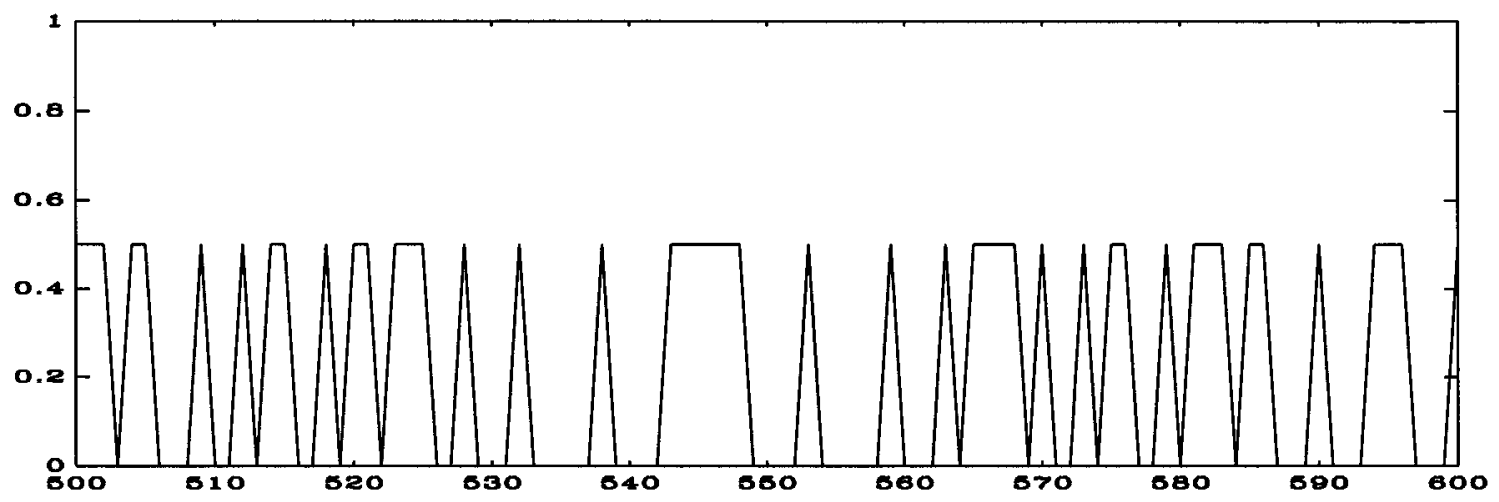

(f)

Fig. 10 Continued.

Finally, the technique proposed by Singh and Joseph ${ }^{12}$ of extracting quantitative information from a binary symbol sequence was also applied. To do so, we first represented our symbol sequence as a string of 1's and -1 's. These values are chosen so that the expected mean of a random sequence of equally likely symbols would be zero. In our case, we changed 0 's to -1 's. An autocorrelation on such a symbol sequence is defined as

$r(n)=\frac{1}{N} \sum_{k=1}^{N} u(k+n) u(k) \quad(n=0,1,2, \cdots, N \gg n)$.
If the sequence is chaotic, the autocorrelation should have the property

$r(n) \rightarrow 0$ as $n \rightarrow \infty$.

We obtained this value only for the chaotic case reported. When some type of periodicity was present, as in Fig. 8, a plot of autocorrelation versus time delay shows peaks with value 1 every 70, 140, or 280 time units. 


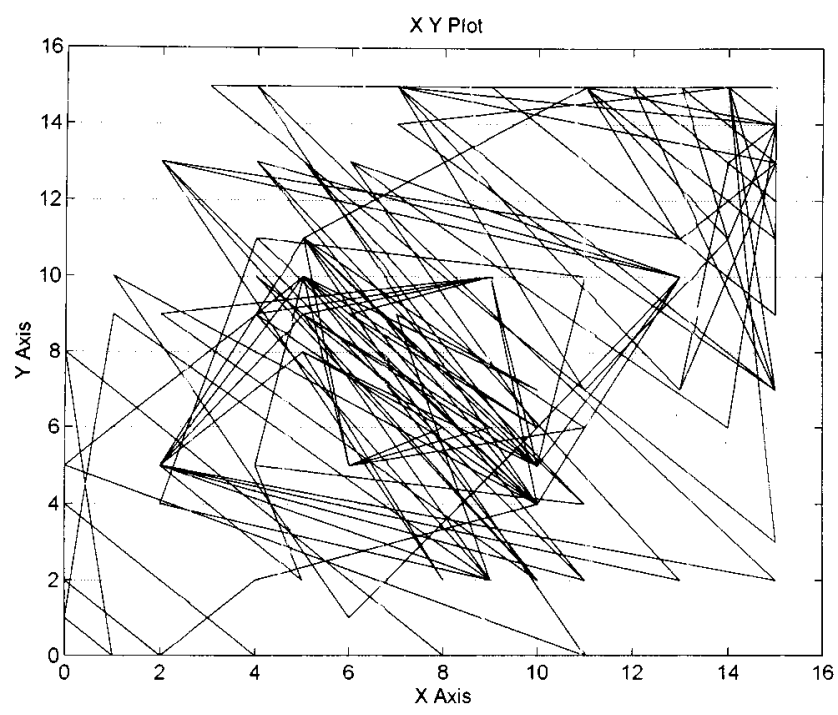

Fig. 11 Diagram $t_{i+1}$ versus $t_{i}$ for a chaotic digital signal as in Fig. 10 after converting from binary to hexadecimal.

\section{Conclusion}

A type of digital chaos has been obtained. The basic structure is an optically programmable digital cell, which we reported previously, as the main block for a possible optical computer. Feedback was applied and internal and external time delays were considered. Although previous results with this cell were experimental, this paper concerns mainly its computer simulation. The obtained results indicate that this system can be employed as a random optical bits generator for use in optical communications. Further studies are needed to determine the characteristics of the obtained signal. From the facts reported here, no clear distinction has been made in our results to differentiate them from true randomness. We have not been able to obtain a way to predict what pattern will occur in an identical repetition of the process starting from the same initial state. Initial parameters were very unpredictable and hence no repetition has been obtained. Some classical symbol sequences, such as the Thue Morse sequence, have some equivalence with our results. However, no close relation has been obtained. Further work needs to be done.

Table 3 LZ complexity for the outputs in Fig. 7.

\begin{tabular}{lcccc}
\hline \hline Output & $n$ & $b$ & $c$ & $c / b$ \\
\hline Period 70 & & & 33 & 0.17 \\
Period 140 & 2048 & 186.2 & 65 & 0.34 \\
Period 280 & & & 129 & 0.68 \\
Chaotic & & & 175 & 0.94 \\
\hline \hline
\end{tabular}

\section{Acknowledgments}

This work was partly supported by the Comisión Interministerial de Ciencia y Technología, CICYT, under grants TIC94-1481-E and TIC95-0118.

\section{References}

1. K. Ikeda, "Multiple-valued stationary state and its instability of the transmitted light by a ring cavity system," Opt. Commun. 30, 257261 (1979).

2. M. Okada and K. Takizawa, "Instability of an electrooptic bistable device with a delayed feedback," IEEE J. Quantum Electron. QE-17, 2135-2140 (1981)

3. A. Neyer and E. Voges, "Dynamics of electrooptic bistable devices with delayed feedback," IEEE J. Quantum Electron. QE-18, 20092015 (1982).

4. J. A. Martín-Pereda and A. González-Marcos, "Optical programmable processing element using optical fibers," in Proc. IEEE Lasers and Electro-Optics Society '92 Conf, pp. 374-375 (1992).

5. A. González-Marcos and J. A. Martín-Pereda, "Quasi-chaotic digital behavior in an optically processing element," Proc. SPIE 2038, 67-77 (1993).

6. A. González-Marcos, "Contribución al estudio de estructuras fotónicas para Computación Optica y análisis de problemas conexos," $\mathrm{PhD}$ Tesis, UPM, Madrid (1993).

7. A. González-Marcos and J. A. Martín-Pereda, "Sequences of bifurcations and transition to chaos in an optically-processing element," in Optical Computing, B. S. Wherrett, Ed., pp. 621-624, IOP, Bristol (1995).

8. B. S. Wherrett, "All-optical computation: a design for tackling a specific physical problem," App. Opt. 24, 2876-2883 (1985).

9. R. G. A. Craig, G. S. Buller, F. A. T. Tooley, S. D. Smith, A. C. Walker, and B. S. Wherrett, "All-optical programmable logic gate," Appl. Opt. 29, 2148-2152 (1990).

10. A. Lempel and J. Ziv, "On the complexity of finite sequences," IEEE Trans. Inform. Theory IT-22, 75-81 (1976).

11. S. N. Rasband, Chaotic Dynamics of Nonlinear Systems, Wiley, New York (1990)

12. R. Singh and D. D. Joseph, "Autoregressive methods for chaos on binary sequences for the Lorentz attractor," Phys. Lett. A 135, 247249 (1989)

Ana González-Marcos received her PhD degree from the Universidad Politécnica de Madrid in 1993. From 1986 to 1989 she was a project manager for CAD tools with a private Spanish firm. She was an assistant professor in the Department of Computer Architectures of the Universidad de Alcalá de Henares during the academic year 1993 to 1994 . She is presently an associate professor in the Depart- 
ment of Computer Sciences of the Universidad Alfonso X el Sabio and a member of the steering group of the North Atlantic Treaty Organization Science for Stability Program. She has been working on optical computing and optical interconnections and her present research interests include neurobiological modeling and neural networks.

José A. Martín-Pereda has been a professor of photonic technology and optical communications at E.T.S. Ingenieros de Telecommunicación, Universidad Politécnica de Madrid (UPM), since 1975.

From 1968 to 1971 he pursued doctoral studies in applied physics, at Colorado State University and received his PhD degree in 1971, from UPM, where he was research vice-rector from 1981 to 1985 From 1986 to 1989 he headed of the Department of Production and Communications Technologies at the General Secretary of the Spanish National Plan for Research and Development. His interests include optical communications, optical computing, and computational neuroscience. He has been a Technical Program Committee member of International Conferences since 1984 (ECOC, IOOC) and was the conference chairman of European Conference on Optical Communication 86. He is a senior member of IEEE, a member of OSA and SPIE, and a foundational member of the Spanish Academy of Engineering. 